Recherches en didactique des langues et des cultures

Les cahiers de l'Acedle

17-2 | 2020

Recherches collaboratives en didactique des langues

\title{
Expériences de recherches-collaboratives au service de l'éducation en situation postcoloniale : enjeux, scientificité et légitimité
}

Claire Colombel-Teuira et Véronique Fillol

\section{OpenEdition}

Édition électronique

URL : https://journals.openedition.org/rdlc/7619

DOI : $10.4000 /$ rdlc.7619

ISSN : 1958-5772

Éditeur

ACEDLE

Référence électronique

Claire Colombel-Teuira et Véronique Fillol, «Expériences de recherches-collaboratives au service de l'éducation en situation postcoloniale : enjeux, scientificité et légitimité », Recherches en didactique des langues et des cultures [En ligne], 17-2 | 2020, mis en ligne le 27 avril 2020, consulté le 17 octobre 2022. URL : http://journals.openedition.org/rdlc/7619 ; DOI : https://doi.org/10.4000/rdlc.7619

Ce document a été généré automatiquement le 17 octobre 2022.

\section{$(9 \Theta$}

Creative Commons - Attribution - Pas d'Utilisation Commerciale - Pas de Modification 4.0 International - CC BY-NC-ND 4.0

https://creativecommons.org/licenses/by-nc-nd/4.0/ 


\title{
Expériences de recherches- collaboratives au service de l'éducation en situation postcoloniale : enjeux, scientificité et légitimité
}

\author{
Claire Colombel-Teuira et Véronique Fillol
}

\section{NOTE DE L'ÉDITEUR}

Avec la contribution de Vahimiti Bousquet, enseignante, maitresse d'accueil, Nouméa $\mathrm{CCT}$ et VF ont collaboré ensemble à plusieurs programmes de recherche sociolinguistique mettant en lumière la complexité des dynamiques de contacts de langues et de cultures. Elles travaillent conjointement à une meilleure contextualisation didactique des enseignements en NC, notamment en créant des outils d'éveil aux langues-cultures océaniennes ou en accompagnant des pratiques innovantes ou des recherches-actions-interventions en contexte scolaire.

1 Sociolinguistes et sociodidacticiennes, nous nous situons dans un contexte formatif où les questions d'éducation plurilingue sont prioritaires et relèvent d'enjeux sociaux, sociétaux et éthiques importants depuis une vingtaine d'années (Colombel 2012 ; Fillol 2012).

2 Nous avons eu la chance de participer ou d'initier plusieurs programmes de recherche ambitieux : première expérimentation sur l'introduction des langues kanak à l'école (Nocus \& al., 2005 ; Salaün, 2005 ; Fillol \& al., 2007), ECOLPOM, 2009-2011 (Nocus \& al. 2014 ; Fillol \& Colombel, 2011, 2014 et 2016; Alby \& al. 2015), Les langues dans la ville (Colombel \& al., 2016; Fillol \& al., 2017a), ou plus «modestes» que l'on pourrait qualifier de recherche-collaborative en didactique du français contextualisé (Colombel- 
Teuira, Fillol \& Bousquet, soumis). Ces expériences nous ont conduites à réfléchir aux enjeux et méthodes de recherche en situation postcoloniale (Fillol \& Le Meur, 2014), et aux conditions de recherches interdisciplinaires (Fillol \& al, 2017b).

En revenant plus précisément sur deux expériences de recherche visant l'inclusion des langues «locales» pour l'une et la prise en compte des usages langagiers dans une approche de type polynomique pour l'autre, nous souhaitons revenir sur les conditions qui rendent possible les recherches collaboratives et les processus de co-construction des savoirs. L'analyse de ces expériences prend en compte trois types de données du terrain: une posture réflexive pour les chercheurs et l'enseignante-chercheure; l'analyse des discours et des gestes professionnels de l'enseignante ; et enfin, l'analyse des indicateurs de réussite ou plus simplement l'observation du développement des compétences littéraciques des élèves.

\section{Faire de la recherche sur les langues, les plurilinguismes et l'éducation plurilingue en contexte sensible}

4 Plusieurs caractéristiques du terrain néo-calédonien participent à définir notre situation en tant que chercheures, formatrices et enseignantes et ont d'une certaine manière orienté nos objets de recherches, nos positionnements épistémologiques et nos conceptions et manières de faire de la recherche.

5 Nous travaillons dans un contexte sensible et paradoxal : sensible, dans le sens où les questions (socio)linguistiques et d'éducation plurilingue sont enchâssées dans des tensions ou consensus politiques (Colombel, 2012; Fillol, 2013; Fillol \& Colombel, 2014) nous incitant à être le plus explicite possible pour ce qui concerne nos intentions et postures de recherche, et paradoxal, dans la mesure où le plurilinguisme qui est la norme de facto est jaugé à l'aune d'une idéologie monolingue en contexte scolaire (Alby \& al., 2015 ; Fillol, 2017 ; Razafimandimbimanana et Favard, 2018).

6 Le compromis politique en matière d'introduction des langues kanak à l'école (Fillol \& Vernaudon, 2004; Fillol, 2013) n'a pas permis d'afficher ouvertement une école inclusive de la diversité linguistique et culturelle des élèves comme c'est le cas en Polynésie française. Comparativement, l'objectif d'une école plurilingue est très explicite, en témoigne la charte de l'Éducation, votée à l'unanimité par les élus de l'Assemblée de la Polynésie française le 29 août 2011, qui précise que :

7 L'objectif de l'École est la réussite de tous les élèves. Cette réussite impose la maitrise du langage qui passe par le développement des compétences linguistiques en français, en langues polynésiennes et en langues étrangères. L'École doit tirer profit de la diversité linguistique ${ }^{1}$ de la société polynésienne pour favoriser le plurilinguisme tout au long de la scolarité.

8 Nous plaçons notre démarche de compréhension et d'analyse des représentations et des pratiques langagières dans le champ de la sociodidactique et de la sociolinguistique scolaire, donc des liens entre langues au pluriel, langues identitaires, langues d'enseignement, tentant de prendre en compte les données sociolinguistiques issues de la situation des apprenants et du cadre scolaire pour les réinvestir dans les situations d'enseignement / apprentissage. Outre la visée sociodidactique, et méthodologique (quels outils d'observation des pratiques langagières et des pratiques enseignantes en 
particulier ?), les recherches que nous souhaitons poursuivre visent à approfondir la réflexion à caractère épistémologique sur les conceptions notamment du bilinguisme/ plurilinguisme, la question de l'évaluation des compétences langagières (Clerc \& Fillol, 2014) des élèves plurilingues, celles de l'enseignement de la lecture/écriture en contexte plurilingue.

9 Nous présentons de façon synthétique une « fiche d'identité » des deux programmes de recherche de façon à envisager les différences relatives aux formes de recherchecollaborative, aux positionnements épistémologiques et éthiques, à l'implication (ou non) des acteurs du terrain et à « définir » les conditions d'une recherche collaborative. L'expérience du programme ANR Ecolpom nous a fait souhaiter, et concrétiser, d'autres formes de recherche, notamment la recherche-action-intervention.

Tableau : comparaison des programmes ECoLPOM et Je lis, j'écris le monde

\begin{tabular}{|c|c|c|}
\hline & ECOLPOM (2009-2012) & Je lis, j'écris le monde (2017-2018) \\
\hline Financement & ANR & Auto-financée \\
\hline $\begin{array}{l}\text { Désignation a posteriori du } \\
\text { programme de recherche }\end{array}$ & $\left(2^{\text {ème }}\right)$ Expérimentation ${ }^{2}$ & Recherche-action-intervention \\
\hline $\begin{array}{l}\text { Point de départ de la } \\
\text { recherche }\end{array}$ & $\begin{array}{l}\text { Question scientifique des } \\
\text { psycholinguistes }\end{array}$ & Besoins du terrain \\
\hline Finalités & $\begin{array}{l}\text { Evaluation de l'introduction des } \\
\text { langues locales sur les compétences } \\
\text { en français }\end{array}$ & $\begin{array}{l}\text { Développer les compétences en } \\
\text { lecture-écriture en français }\end{array}$ \\
\hline Acteurs & $\begin{array}{l}\text { Chercheurs (de différents champs } \\
\text { disciplinaires) et institutions }\end{array}$ & $\begin{array}{l}\text { Enseignante, chercheur-e-s, } \\
\text { parents, élèves }\end{array}$ \\
\hline Relation entre les acteurs & $\begin{array}{l}\text { Rapport de domination réel ou } \\
\text { symbolique }\end{array}$ & $\begin{array}{l}\text { Relation d'équité des rôles et des } \\
\text { expériences }\end{array}$ \\
\hline Forme de recherche & $\begin{array}{l}\text { Recherche pluridisciplinaire et } \\
\text { dimension comparée }\end{array}$ & Recherche-action-intervention \\
\hline $\begin{array}{l}\text { Références théoriques } \\
\text { partagées entre les } \\
\text { chercheur.e.s }\end{array}$ & $\begin{array}{l}\text { Partiellement (travaux de J. } \\
\text { Cummins notamment) }\end{array}$ & Totalement \\
\hline Posture des acteurs & Non associés ou impliqués & $\begin{array}{l}\text { Enseignante impliquée, } \\
\text { "praticien réflexif» (Schön, } \\
\text { 1994) et posture engagée pour } \\
\text { le/la chercheur-e }\end{array}$ \\
\hline Niveau & Recherche macro & Recherche micro-contextualisée \\
\hline Méthodologie $^{3}$ & Quantitative et qualitative & $\begin{array}{l}\text { Démarche collaborative et } \\
\text { interprétative }\end{array}$ \\
\hline
\end{tabular}




\begin{tabular}{|l|l|l|}
\hline Vision de la recherche & «Top-down» & «grounded » ou située \\
\hline
\end{tabular}

10 Le programme École Plurilingue Outre-mer всоцром (2009-2012) qui s'inscrivait dans la continuité d'un séminaire (2007) et d'une première "évaluation» (voir note 2) constituait pour nous - chercheures en sociodidactique impliquées dans le contexte calédonien - l'occasion d'une recherche collaborative.

L'originalité de ces dispositifs est qu'ils ont dès le départ visé l'association des chercheurs (linguistes, sociolinguistes, psychologues, anthropologues) et des professionnels de l'éducation chargés de leur mise en œuvre (enseignants, conseillers pédagogiques, inspecteurs, autorités pédagogiques locales, etc.). Cette association devait répondre à un triple objectif: mettre la recherche au service d'une volonté institutionnelle locale; participer au rééquilibrage des rapports de domination hérités de la période coloniale, notamment en faveur des langues marginalisées dans un contexte de diglossie; promouvoir une recherche collaborative impliquant chercheurs et acteurs de terrain. (Salaün \& Vernaudon, 2014: 159)

11 Au-delà des difficultés de la collaboration inter ou pluridisciplinaire ${ }^{4}$ qui auraient mérité d'être discutées tout au long du programme dans une démarche réflexive et/ou critique $^{5}$, quel est le point de départ de la recherche? Qui pose la question de recherche ${ }^{6}$ ? L'ensemble des acteurs impliqués validaient-ils la question? La demande est-elle sociale ou politique? (Clerc \& Fillol, 2014).

Une des premières questions que le chercheur engagé peut se poser - et selon nous qu'il a à se poser lorsqu'il s'engage dans une recherche financée - est celle-ci : pour répondre à quels problèmes sociaux et posés par qui ? Ou, autrement formulée : à quelle demande et émanant de qui ? (...) le chercheur doit-il attendre l'émergence d'une demande sociale? Peut-il, doit-il la susciter, aider ou accompagner son émergence quand elle est latente, non clairement exprimée ? (Clerc et Fillol, 2014)

La recherche-action (démarche que nous privilégions) partage avec l'expérimentation quelques étapes mais aussi de nombreuses différences : le questionnement, le problème à résoudre, la formulation d'une hypothèse, la mise à l'épreuve de l'hypothèse ne sont pas énoncés ou imposés par le chercheur mais co-construits avec l'ensemble des acteurs impliqués (Clerc, 2015).

\section{Conditions d'une recherche-action-intervention et implication}

13 Pour le projet je lis, j'écris le monde, le point de départ est bien une demande de collaboration pédagogique et scientifique, celle d'une enseignante de $\mathrm{CP}$ en zone prioritaire, Vahimiti Bousquet adressée à Claire Colombel-Teuira, enseignante chercheure qui a développé des outils d'éveil aux langues océaniennes. C'est à partir d'une part, du constat des difficultés des élèves à entrer dans le code écrit, et d'autre part, de ses gestes professionnels qualifiés selon elle d'« épuisés » qu'est née cette recherche-action en éducation définie comme :

une pratique méthodologique centrée sur la résolution d'un problème concret vécu dans une situation pédagogique réelle dans le but d'y apporter des changements bénéfiques, de contribuer au développement professionnel des personnes qui y ont part et d'améliorer les connaissances sur cette situation (Guay et Prud'homme, $2011: 188)$ 
14

int essentiel qui découle du choix de recherche (celui de la question de recherche imposée ou négociée, ou d'un problème concret à résoudre) concerne la relation entre enseignants, acteurs impliqués et chercheurs. Si, dans le cadre du programme ECOLPOM ${ }^{7}$, nous avons pu approfondir la connaissance et la compréhension des rapports aux langues, aux normes, aux pratiques plurilingues, dessiner une cartographie des "micro-contextes ${ }^{8}$ et suggérer des pistes de dispositifs inclusifs des langues océaniennes à l'école calédonienne (Fillol \& Colombel, 2014 et 2016), la perception de notre rôle ou place dans la recherche en tant que "chercheurs prescripteurs » et « évaluateurs » n'a pas permis de co-construire des outils pédagogiques ou d'envisager des recherches-actions-interventions qui partent des problèmes ${ }^{9}$ ou questions posés par les acteurs dans les écoles et leurs classes. Pour le dire simplement, cette recherche a été perçue comme une recherche par et pour les chercheurs, qui au final perturbait le quotidien de la classe.

Pour Dewey, souvent considéré comme le précurseur de la recherche-action en éducation, les généralisations de chercheurs extérieurs à l'école sont rarement réinvesties par les enseignants et donc insuffisantes pour améliorer nos systèmes d'éducation: "la connaissance trouve sa source dans l'action collaborative des chercheurs et des praticiens et des praticiens sur le terrain » (Karsenti et Savoie-Zajc, $2011: 185)$. différents niveaux, l'un que l'on pourrait qualifier de « pratique » ou " pédagogique ", à savoir accompagner l'enseignante à faire entrer les élèves dans le code écrit, dans la conceptualisation de l'écrit et très spécifiquement le principe alphabétique; l'autre niveau est d'ordre scientifique ou didactique. En effet, « plusieurs travaux sociologiques et sociolinguistiques, analysent la réussite dans l'apprentissage du lire-écrire d'élèves de milieux sociaux contrastés par la faculté de mise à distance du langage afin de l'appréhender en tant qu'objet dans un processus de "secondarisation » qui caractérise la littéracie scolaire. » (Miguel-Addisu et M.-O. Maire-Sandoz, 2018 :17). L'hypothèse et « l'originalité de ce projet d'éveil à la diversité est de se servir des langues autres que celle de l'école pour mieux apprendre à lire et écrire » (Ina Pepari, 2019 : 9). le monde - est avant tout une question de relation humaine. Outre la relation de confiance et de respect mutuels, une très grande disponibilité hors-temps scolaire ou formatif sont autant de facilitateurs de la réussite du projet (et de sa valorisation). On peut parler dès lors de véritable implication de l'enseignante et des chercheur-e-s. « La R.A ne se contente pas d'être impliquée parce que qu'elle se situe sur le terrain, elle est impliquée par immersion dans un temps long »(Clerc, 2015 : 119), "l'au-delà de cette implication du chercheur, déjà en soi importante, est l'action sur, pour et avec le terrain dans une perspective de (trans)formation ». (ibid)

18 Au-delà des chercheurs et enseignants, d'autres acteurs devraient avoir une place plus explicite dans les recherches en éducation. Les parents d'élèves sont rarement impliqués dans les projets didactiques. Pour des raisons socio-historiques (Colombel, 2012), l'école est une institution dont on se méfie. Le rapprochement famille-école était aussi une question investiguée par les programmes "expérimentation de l'enseignement des LK (2002-2004)»(Fillol al., 2007) et le programme EColPOM. Ces projets ont souligné combien la demande d'une école plurilingue était forte (Fillol et Colombel, 2014). Il est l'un des enjeux du projet je lis, j'écris le monde, mais aussi un 
facteur de réussite. En effet, certains parents ont exprimé un sentiment de fierté d'être sollicités en tant "qu'experts" par l'enseignante. La légitimation des langues identitaires des enfants est une sorte de médiateur facilitant la coéducation.

Dans le contexte sensible de la Nouvelle-Calédonie, où certains acteurs (enseignants, parents d'élèves) se sentent traités comme des « cobayes » par les chercheurs, il s'agit pour nous de créer un espace de légitimation du "savoir d'expérience» (Schön, 1994) en valorisant, dans une approche réflexive, la «compétence d'acteur en contexte " (Giddens, 1987).

La représentation initiale que chaque acteur se faisait de la complémentarité dans la co-construction du savoir est l'objet d'une négociation dans l'interaction, négociation notamment influencée par le rapport plus égalitariste proposé par la chercheuse qui sollicite le point de vue réflexif des praticiennes. (Morrissette et Desgagné, 2009: 123)

En effet, dans notre pratique, c'est cet espace de négociation qui crée la «zone interprétative " (Davidson, Wasser et Bresler, 1996) des situations d'enseignementapprentissage. La co-élaboration d'un cadre d'action partant des besoins des praticiens nous semble en conséquence, incontournable afin de produire un travail de recherche éclairant les pratiques, les gestes professionnels, les postures et stratégies des élèves mais aussi les enjeux didactiques pour une école inclusive. À ce titre nous faisons nôtres les mots de Prud'homme, Presseau et Dolbec :

Il nous apparaissait primordial d'opter pour des études en profondeur du particulier et de la complexité afin d'agir sur les difficultés dans le contexte de la pratique, au regard de la mobilisation des connaissances issues de la recherche. (2007:39)

21 Pour travailler spécifiquement les identifications et changements de postures, nous nous sommes largement inspirées de l'utilisation de l'autoscopie conçue par C. Colombel (2012) dans le cadre de sa thèse, "technique» de formation "permettant l'analyse réflexive des praticiens à travers l'auto-observation décentrée » (Colombel, 2012 : 101).

Les références théoriques conjointes (en particulier le paradigme de l'agir professionnel de Jorro ou de l'agir enseignant de Bucheton), et notre commune conception socio-constructiviste de l'enseignement/apprentissage, ont aussi facilité la réalisation du projet de bout en bout. Les regards croisés ${ }^{11}$ d'une praticienne experte et d'une sociodidacticienne ont permis de construire et réajuster le projet au fur et à mesure pour arriver à une version aboutie, sa mise en œuvre et son analyse.

\section{Pour quels « résultats » ou quels « effets »?}

Les effets de ces deux programmes sur les différents acteurs de l'éducation nous semblent corrélés à l'implication ressentie ou aux espaces discursifs/interprétatifs accordés.

Le projet Je lis, j'écris le monde a été pensé, construit et réajusté pour répondre aux besoins des élèves identifiés par l'enseignante. Les effets sur les performances et attitudes des élèves, au-delà d'être attendus constituaient l'objectif même du projet. D'un point de vue méthodologique, nos outils de mesure des performances des élèves ont d'abord été les outils d'évaluation de la maitresse et les tests OURA-LEC ${ }^{12}$. Selon ces indicateurs institutionnels, tous les élèves ont progressé et ont atteint au minimum un 
degré satisfaisant de maitrise de la combinatoire. Les observations et films de classe ont aussi permis d'identifier et de quantifier des performances ou attitudes plus rarement évalués en classe. Nous avons ainsi noté une amélioration générale, mais singulièrement importante pour les élèves réputés en difficulté, dans l'engagement et la persévérance dans la tâche ${ }^{13}$ (Colombel-Teuira, 2019). Lors d'entretiens (semi-dirigés) avec les élèves (seul ou en groupe), nous avons aussi pu relever des indices de l'augmentation de leur appétence à la lecture et du développement de leurs habiletés métalangagières et métacognitives. Par exemple, lors d'un bilan de fin d'atelier de manipulation lettres/syllabes, un élève s'est écrié « ah mais, mais maitresse, j'ai compris. Ben mon piège à moi c'est à cause que je me trompe des lettres et des syllabes que c'est pas pareil ${ }^{14}$.»

25 Du point de vue des enseignants, les deux projets ont eu des résonnances très différentes tant sur les « sentiments » et représentations liés au déroulement que sur le développement de compétences professionnelles. D’un point de vue méthodologique, notons cependant que le suivi et l'accompagnement réflexif n'a pas été équivalent. Pour le projet ЕсоцРом, certains enseignants n'ont pas accepté d'être filmés, d'autres n'ont accepté que de répondre à des questionnaires auto-administrés et aucun n'a tenu de carnet de bord ${ }^{15}$.

Dans le cadre de la collaboration avec Vahimiti Bousquet, les modalités de l'accompagnement ayant été négociées et conçues conjointement, les différentes techniques et outils proposés ont pu être mis en place. Le carnet de bord, par exemple, s'est révélé plus un outil de distanciation pour la praticienne ${ }^{16}$ qu'un mode de suivi pour les chercheures. Vahimiti Bousquet témoigne dans un portfolio :

Au-delà des progrès des élèves, qui sont tous entrés dans la combinatoire à la fin du projet, j'ai moi aussi progressé dans l'analyse de mes gestes professionnels. Sans prétendre à l'expertise, je pense maintenant avoir développé de nouvelles compétences pour accompagner des collègues, enseignants du premier degré. (Bousquet, $2018: 27$ )

Les effets en termes de recherche-action-formation ne sont pas négligeables. En effet, les différents projets pédagogiques et recherches-actions engagées visant à valoriser les identités des élèves et l'engagement dans la littéracie (Cummins, 2011) lui ont permis de construire des compétences professionnelles pour l'accompagnement de jeunes enseignants autour de l'observation multi-modale ${ }^{17}$ comme moyen d'identifier les besoins des élèves et de les évaluer autrement. Enfin, l'action réflexive et le travail collaboratif ont contribué à rassurer l'enseignante, "à légitimer son action pédagogique et sa réflexion didactique. Elle s'autorise en effet, à développer une éducation au plurilinguisme - non légitimée - dans les programmes scolaires calédoniens » (Colombel-Teuira et al., soumis).

Pour les chercheur-e-s, notre collaboration dans le cadre du programme ECOLPOM a été fructueuse du point de vue de la comparaison entre les dispositifs d'enseignement et les représentations du plurilinguisme en milieu scolaire (dans les différentes collectivités), du point de vue méthodologique, sur le plan relationnel avec les chercheurs en sociolinguistique ou ethno ou anthropolinguistique des autres territoires. Nous avons interrogé le choix des langues enseignées, la place qui leur est octroyée à l'école, les formes d'enseignement proposées au détriment cependant de co-construction de savoirs sur l'enseignement bilingue ou la bi-littéracie. À l'inverse, le projet je lis, j'écris le monde, a permis une description compréhensive de l'apprentissage de la lecture en contexte plurilingue. Les enjeux sont d'augmenter les connaissances scientifiques en 
matière d'enseignement-apprentissage en contexte plurilingue (lire c'est utiliser un code arbitraire qui transcrit arbitrairement un code arbitraire) et plus spécifiquement comprendre les mécanismes à l'œuvre dans les situations d'apprentissage.

\section{Pour conclure}

Les deux recherches présentées illustrent bien les tensions habituelles entre les différents courants en recherche en éducation (ou plus largement en SHS) qui opposent parfois scientificité/éthique, "hors-sol»/impliqué. Ces types de recherches sont complémentaires en matière de production de savoirs comme en termes d'enjeux sociaux, mais très différents en termes de positionnement et d'implication : faire de la recherche qualitative implique finalement de remplacer les concepts de "quantité, objectivité, neutralité, généralisation, représentativité", par ceux de "qualité, pertinence, cohérence, argumentation, rigueur, appropriation, sens et sujet " (Vatz Laaroussi, 2007 : 11).

Dans le cas de la Nouvelle-Calédonie, le projet ECoLPOM avec la duplication de résultats internationaux participe à la déconstruction de stéréotypes, hérités de la période coloniale (Colombel, 2012). Si on se positionne dans une optique de formation des enseignants, il semblerait que les recherches implicantes, comme le projet Je lis, j'écris le monde, favorisent le changement à long terme (cf. les travaux de K. Lewin dans les années 1940 sur la dynamique de groupe).

31 Une des finalités majeures de nos recherches en sociodidactique est de contribuer au développement d'une perspective plurilinguistique pour une école plus inclusive des pratiques langagières des élèves (Clerc et Fillol, 2014). Nous pensons que l'éveil aux langues à l'école calédonienne peut être un moyen de valoriser les plurilinguismes, de légitimer les approches inclusives des enseignants et d'envisager d'autres moyens d'entrer dans la lecture. L'ambition de ces projets est aussi de contribuer non seulement à la décolonisation des savoirs concernant les dynamiques plurilingues en contexte scolaire mais plus encore à l'émancipation des outils de l'école (Razafimandimbimanana \& Favard, $2018: 331$ ). Ainsi, si plusieurs enseignants étaient concernés dans le programme Ecolpom et une seule pour je lis, j'écris le monde, paradoxalement, seule V. Bousquet, désormais maitresse-formatrice, contribue par son action collaborative avec les enseignants de sa circonscription à une diffusion à plus grande échelle de la réflexion didactique co-construite, avec comme outils et compétences : une meilleure conceptualisation de la lecture-écriture (en contexte dit sensible), une ingénierie pour la pédagogie différenciée et le renforcement de son identité professionnelle.

Nous faisons le constat de l'échec d'une transposition didactique qui ferait/fait le trajet savoir savant, décideur institution et classe-société (autrement dit l'échec du modèle " applicationniste ») pour d'autres formes de recherche en éducation, en particulier, la recherche-collaborative sur le principe d'équité des savoirs et partages d'expériences par la mise en commun de valeurs, de concepts, de connaissances, d'analyses, et la coconstruction d'outils pour la classe.

Vatz Laaroussi, M. (2007). La recherche interculturelle : une recherche engagée ? Recherches qualitatives, Hors-Série, $\mathrm{n}^{\circ}$ 4, 2-13. 


\section{BIBLIOGRAPHIE}

Alby S., Colombel C., Fillol V., et Leglise I. (2015). L'école française face aux plurilinguismes de l'Outre-mer. Dans de Pietro, J.-F. et Rispail, M., L'enseignement du français à l'heure du plurilinguisme. Vers une didactique contextualisée. Namur : AIRDF et Presses Universitaires de Namur, Collection « Recherches en didactique du français ", n 6, 89-104.

Bousquet, V., (2018). Sur le chemin de l'école : à la croisée des parcours linguistiques et culturels des enseignants et des élèves, Portfolio DU Fles-Interculturalités. Nouméa, Université de la NouvelleCalédonie.

Bucheton, D. (2009). L'agir enseignant : des gestes professionnels ajustés. Toulouse, Octarès Editions.

Clerc, S. (2015). La recherche-action : ancrages épistémologique, méthodologique et éthique. Dans Blanchet, Ph. et Chardenet, P. (dir.), Guide pour la recherche en didactique des langues et des cultures. Approches contextualisées. Paris, Éditions des Archives Contemporaines \& Agence Universitaire de la Francophonie, 112-121.

Clerc, S. Fillol, V. (2014). Plaidoyer pour des recherches en sociodidactique émancipées du paradigme de l'évaluation. Dans Ottavi, P. et Di Miglio A. (dir.), Faire société dans un cadre pluriculturel. Limoges, Lambert Lucas, 181-190.

Colombel C., (2012). Langues kanak, français, langues d'enseignement et de culture en NouvelleCalédonie : quelle glottopolitique pour quelle contextualisation sociodidactique? Thèse de doctorat, Université d'Aix-Marseille.

Colombel-Teuira C. (2019). « Je lis, j’écris le monde » : Développer des compétences littéraciques des élèves avec les langues océaniennes, communication dans le cadre du COOL 11, 8 octobre 2019. Nouméa, Université de la Nouvelle-Calédonie.

Colombel-Teuira, C. et Fillol, V. avec la collaboration de Bousquet, V. (sous presse) Observer et objectiver le « déjà-là » des élèves : premiers pas vers la bienveillance linguistique ? Dans Dinvaut, A. et Bichlë, L. (dirs.), Mieux vivre en langues : la bientraitance linguistique. Avignon, Editions de l'université d'Avignon.

Colombel-Teuira C., Fillol V., Geneix-Rabault S., Vandeputte-Tavo, L. (dirs.) (2016). Les langues dans la ville : pratiques plurilingues et artistiques à Nouméa. Rapport de recherche, Nouméa, UNCDGLFLF.

Cummins, J. (2011). De l'importance des données de la recherche empirique pour les politiques éducatives en faveur des apprenants en difficulté. Division des politiques linguistiques, direction de l'éducation et des langues, DGIV, Conseil de l'Europe, Strasbourg. Disponible à : www.coe.int/lang/fr.

Davidson Wasser, J. et Bresler, L. (1996). Working in Interpretive Zone: Conceptualizing Collaboration in Qualitative Research Teams. Educational Researchers, vol. 25, n 5,1996,5-15.

Fillol, V. (2017). Les langues océaniennes et le-s plurilinguisme-s. Études en Didactique des langues, $n^{\circ} 28,137-150$.

Fillol, V. (2013). Les langues kanak ont-elles manqué leur (r)entrée à l'école calédonienne ? Dans Becetti, A., Blanchet, P. et Colonna, R., Politiques linguistiques et plurilinguismes. Du terrain à l'action glottopolitique. Paris, L’Harmattan, Collection Espaces discursifs, 49-68. 
Fillol, V. (2012). « Enjeux d'une sociodidactique des langues d'enseignement et de culture en Nouvelle-Calédonie ». Rispail, M. (dir.), Jeannot, C., Tomc, S. et Totozani, M. (eds), Esquisses pour une école plurilingue. Réflexions sociodidactiques. Paris, L'Harmattan, 77-97.

Fillol, V. et Colombel, C. (2016). Langue française et cultures océaniennes : quelle éducation plurilingue pour la Nouvelle-Calédonie ? Dans Hélot, C. \& Erfurt, J. (eds) : L'éducation bilingue en France : politiques linguistiques, modèles et pratiques. Rennes, Presses Universitaires de Rennes, 118-129.

Fillol, V. et Colombel, C. (2014). Quelles politiques linguistiques éducatives pour la NouvelleCalédonie ? Quels dispositifs d'enseignement des langues ? Réflexions sociodidactiques. Dans Nocus, I., Vernaudon, J. et Paia, M. (dir.), Apprendre plusieurs langues, plusieurs langues pour apprendre. L'école plurilingue en Outre-mer. Rennes, Presses Universitaires de Rennes, Collection « Des sociétés », 199-221.

Fillol, V. et Colombel, C. (2011). Quelles places pour les langues des élèves à l'école calédonienne? Repères sociolinguistiques pour l'enseignement des langues en Nouvelle-Calédonie. Rapport de recherche final du Programme всоцом, programme financé par l'Agence Nationale de la Recherche, non publié.

Fillol, V., Colombel-Teuira, C., Geneix-Rabault, S., Vandeputte-Tavo, L. (2017a). Les langues dans la ville de Nouméa : sociolinguistique urbaine et interdisciplinarité. Pour une recherche à visée sociale en contexte postcolonial. Dans SOCLES La sociolinguistique urbaine pour penser les villes, en Algérie et dans d'autres contextes plurilingues... Hommage au travail de Thierry Bulot, Numéro spécial, janvier. Disponible à : https://www.asjp.cerist.dz/en/Articles/25.

Fillol, V., Geneix-Rabault, S., Vandeputte-Tavo, L. et Colombel-Teuira, C. (2017b). Vivre et partager son terrain : retour sur un programme de recherche interdisciplinaire Les Langues dans la ville (Nouméa). Communication au Congrès international RFS Identités, conflits et interventions sociolinguistiques, Université Paul Valéry, 15 juin 2017.

Fillol, V. et Le Meur, P.-Y. (dir). (2014). Terrains océaniens : Enjeux et méthodes. Paris, L'Harmattan, Collection Cahiers du Pacifique Sud Contemporain.

Fillol V., Vernaudon J., Salaün M. et Nocus, I., (2007). L'école républicaine française et les langues kanak. Dans Burban, C. et Lagarde C., (travaux réunis par), L'école, instrument de sauvegarde des langues menacées? Presses Universitaires de Perpignan, Collection Etudes, 295-307.

Fillol, V. et Vernaudon, J. (2004). Les langues kanak et le français, langues d'enseignement et de culture en Nouvelle-Calédonie : d'un compromis à un bilinguisme équilibré ? ELA, n 133, Paris, 55-67.

Karsenti, T. et Savoie-Zajc, L. (2011). La recherche en éducation : étapes et approches. Editions du renouveau pédagogique, $3^{\mathrm{e}}$ édition.

Giddens, A. (1987). La constitution de la société. Paris, Presses universitaires de France.

Guay, M.-H. et Prud'homme, L., (2011), « La recherche-action ». Dans Karsenti, T. et Savoie-Zajc, L., La recherche en éducation : étapes et approches. Editions du renouveau pédagogique, 3e édition, 184-211.

Pepari, I. (2019). Je lis, j'écris le monde. Publication indépendante.

Jorro, A. (2002). Professionnaliser le métier d'enseignant. Paris, ESF.

Miguel-Addisu V. et Maire-Sandoz M.O. (2018). Enseigner la lecture dans une classe plurilingue et défavorisée. Carrefours de l'éducation, n 46, 15-26. 
Morrissette, J. et Desgagné, S. (2009). Le jeu des positions de savoir en recherche collaborative : une analyse des points de vue négociés d'un groupe d'enseignantes du primaire. Dans Approches inductives II, Recherches Qualitatives $n^{\circ} 28,118-144$.

Nocus, I., Vernaudon, J. et Paia, M. (2014). Apprendre plusieurs langues, plusieurs langues pour apprendre. L'école plurilingue en Outre-mer. Rennes, Presses Universitaires de Rennes, Collection « Des sociétés».

Nocus, I., Pineau-Salaün, M., Fillol, V. et Vernaudon, J. (2007). La prise en compte des langues maternelles kanak favorise-t-elle l'appropriation du français ? Bilan d'étape d'une expérimentation en Nouvelle-Calédonie. Dans Noyau, C. (dir.) Actes du colloque « Appropriation du français et construction de connaissances en situation diglossique », 24-25 février 2005, éd. Paris X, document multimédia sur cédérom.

Nocus, I. Florin, A., Guimard, P. (2005). Rapport d'évaluation de l'expérimentation « L'enseignement des langues et de la culture kanak à l'école primaire de la Nouvelle-Calédonie ». Axe psycholinguistique, Laboratoire de Psychologie « Education, cognition, développement » (Labécd), Université de Nantes (non publié).

Nusbaum, L. (2008). Construire le plurilinguisme à l'école : de la recherche à l'intervention et de l'intervention à la recherche. Dans Candelier, M., Ioannitou, G., Omer, D. et Vasseur M.-T. ( dir.), Conscience du plurilinguisme, Presses Universitaires de Rennes, 125-144.

Prud'homme, L., Presseau, A. et Dolbec, A. (2007). Une pratique réflexive de recherche 'à trait d'union' : quels enjeux scientifiques? Dans Actes du colloque Approches qualitatives et recherche interculturelle : bien comprendre pour mieux intervenir, Recherches Qualitatives, hors-série $n^{\circ} 4,36-68$.

Razafimandimbimanana, E. et Favard, N. (2018). Les élèves aux besoins éducatifs particuliers se mettent en représentation : « on parle plusieurs langues pour progresser ». Dans Enseignement et formation du/en français en contexte plurilingue. Hanoï, Edition de l'Université nationale du Vietnam à Hanoï, 311-335.

Salaün, M. Vernaudon, J. (2014). Décoloniser la recherche, pratiquer la recherche en contexte de décolonisation : retours sur la promotion d'une école plurilingue. Nouvelle-Calédonie, Polynésie française, 2002-2012. Dans Fillol, V. et Le Meur P.-Y. (dir.), Terrains océaniens : enjeux et méthodes. Paris, L'Harmattan, 159-174.

Salaün, M. (2005). Rapport d'évaluation de l'expérimentation «L'enseignement des langues et de la culture kanak à l'école primaire publique de la Nouvelle-Calédonie », Axe sociolinguistique. Département des Sciences de l'Education, Université Paris 5 (non publié).

Schön, D.A. (1994). Le praticien réflexif. Montréal, Les éditions Logiques.

\section{NOTES}

1. C'est nous qui soulignons. On ne trouve aucun équivalent dans l'ensemble du corpus de textes institutionnels calédoniens. Le Projet Educatif de la Nouvelle-Calédonie (PENC) à titre d'exemple comprend trois occurrences du terme "diversité ", à propos de diversité des publics : https:// denc.gouv.nc/textes-de-reference/le-projet-educatif-de-la-nouvelle-caledonie.

2. 2ème expérimentation pour souligner la continuité avec la première «expérimentation de l'introduction des langues kanak à l'école» (titre officiel) mise en œuvre sous la direction scientifique de C. Lercari (2002 à 2004) et financée par le Gouvernement de la Nouvelle-Calédonie avec une évaluation confiée à des chercheures extérieures à la Nouvelle-Calédonie (Salaün, 2005 ; Nocus et al., 2005). 
3. La méthodologie de recherche de l'enquête sociolinguistique en NC est explicitée dans Fillol \& Colombel, 2011, 2014 et 2016. Pour l'ensemble des travaux et "résultats " produits, on se reportera à l'ouvrage: Nocus, I., Vernaudon, J. et Paia, M., 2014, Apprendre plusieurs langues, plusieurs langues pour apprendre. L'école plurilingue en Outre-mer, Presses Universitaires de Rennes, Collection « Des sociétés ».

4. Nous affirmons volontiers aujourd'hui (avec le recul et la réflexivité) que les axes psycholinguistique et sociolinguistique sont moins complémentaires que nous ne le postulions, en ce sens qu'ils s'inscrivent dans des paradigmes épistémologiques éloignés, voire incompatibles, en particulier, en ce qui concerne les conceptions du bi/plurilinguisme.

5. Plusieurs retours sur expériences ou analyses critiques ont été publiés : Clerc et Fillol, 2014 ; Salaün et Vernaudon, 2014.

6. Dans le cadre de la première « expérimentation » de l'introduction des LK à l'école (2002-2004), la question de recherche initiale était explicitement la suivante : « la prise en compte des langues maternelles favorise-t-elle l'appropriation du français?» (Nocus \& al. 2007). Marie Salaün et Jacques Venraudon expliquent avoir ainsi eu la difficulté, voire l'impossibilité de «devoir répondre à une question dont nous ne validions pas les termes " (Muni Toke cité par Salaün \& Vernaudon, $2014: 169)$. Nous faisons pour notre part le constat d'un malentendu dans le sens où une partie des chercheurs cherchait à répondre à une (fausse) demande sociale du groupe dominant quand l'autre partie des chercheurs se positionnait davantage dans une rechercheaction visant à mieux définir les obstacles à une réforme du système scolaire (Clerc et Fillol, 2014).

7. Nous précisons que les réflexions et analyses psycholinguistiques et sociolinguistiques concernant les trois collectivités concernées ont été publiées notamment et pas exclusivement dans : Nocus, I., Vernaudon, J. et Paia, M., 2014, Apprendre plusieurs langues, plusieurs langues pour apprendre. L'école plurilingue en Outre-mer, Presses Universitaires de Rennes, Collection «Des sociétés ».

8. Lifou (Province des Iles) où les élèves sont bilingues drehu-français; Houaïlou (Province Nord) où le multilinguisme est plus complexe et Nouméa (Province Sud) où les élèves ont des répertoires langagiers divers dans un environnement plurilingue à la langue dominante unique (le français).

9. Quel temps consacrer à la lecture ? Comment mener des activités méta-phonologiques en utilisant les deux langues du répertoire des élèves? (Fillol et Colombel, 2011).

10. Ce projet transdisciplinaire utilise des langues (non) parlées par les élèves pour travailler l'apprentissage de la lecture. Les outils construits permettent aux élèves de se focaliser sur une seule tâche pour en automatiser les mécanismes et ensuite pouvoir passer à des tâches complexes que sont la lecture (compréhension) ou l'écriture (orthographique). Dans une perspective actionnelle, la mise en projet s'est faite avec une activité brise-glace, un jeu des familles et des lectures offertes d'albums bilingues (français/inuktitut). Le jeu a été construit en sélectionnant certaines des langues familiales (déclarées par les parents, ajië, cèmuhî, drehu, futunien, tahitien, wallisien et xârâcùù) ainsi que 2 langues du sud-est asiatique pour leurs caractéristiques phonologiques et «orthographiques » (vietnamien et chinois). Les « outils » et la démarche du projet ont été publiés : Pepari I., Je lis, j'écris le monde, 2019 autopublié, ISBN 978-1-7932-3509-1.

11. Le dispositif de recherche s'organise en 5 temps forts itératifs. À partir des questionnements de VB i), nous co-construisons un projet/une séquence ; ii) ensuite, l'enseignante rédige seule ses outils de pilotage, au besoin nous co-élaborons le matériel didactique (affichage, corpus de documents, jeux et manipulations...) ; iii) les séances sont filmées avec parfois aussi des prises de vue simples; iv) nous faisons un premier retour sur pratique en fin de séance, essentiellement à travers des jeux de questions et $v$ ) un deuxième retour sur pratique en nous appuyant sur les captations (vidéos et photographies) nous permet de construire une analyse négociée (Colombel \& Fillol, soumis). 
12. Utilisés dans la circonscription, outils du laboratoire Cognisciences, 2013, http:// www.cognisciences.com/accueil/outils/article/oura-lec-cp-outil-enseignant.

13. Un élève qui « tenait à peine en place plus de 5 minutes » a réussi à augmenter son temps de concentration/réalisation à $21: 25$ minutes.

14. En manipulant un tableau à double entrées pour décoder l'alphabet syllabique inuktitut, cet élève s'est rendu compte que lors qu'il écrivait (en français), il faisait comme si une lettre transcrivait systématiquement une syllabe.

15. Ils rédigeaient bien leurs outils de pilotage, mais ils n'ont transmis aucune progression/ programmation et n'ont pas tenu de carnet de bord spécifique au projet, bien que cela leur ait été proposé.

16. VB présentait le CAFIPEMF et disait avoir des difficultés « à rédiger ». Nous avons alors mis en place le carnet de bord avec des objectifs ciblés évoluant de semaine en semaine pour arriver à l'identification des réajustements des gestes, leur justification et une analyse par rapport aux outils de pilotage (l'enseignante rédigeait toutes les fiches séquences et séances du projet, en plus du cahier journal).

17. Bousquet, V. (2019). Comment aider les enseignants de maternelle à développer une posture d'observateur multimodale pour mieux identifier les besoins des élèves? Mémoire CAFIPEMF.

\section{RÉSUMÉS}

En revenant sur deux programmes de recherche (d'ampleur très différente) concernant l'inclusion des langues océaniennes dans le contexte diglossique calédonien, nous proposons d'expliciter et d'analyser les processus et les implications de la co-élaboration des savoirs. Cette comparaison soulève et éclaire des questions épistémologiques et éthiques. La recherche-action en éducation ne peut se construire que sous la forme d'un travail coopératif entre enseignants en formation, enseignants en exercice et enseignants-chercheurs appartenant à des champs disciplinaires divers. Cependant, le rapport entre équipes de recherche et personnel enseignant est toujours un équilibre fragile (Nusbaum, 2008 : 127). De plus, l'activité de recherche constitue un acte d'exercice de pouvoir, dans ce sens qu'elle construit des réalités sociales et les légitime à travers sa vulgarisation parfois sans l'accord des individus. La recherche-action-intervention nous paraît donc plus efficiente sur le plan éthique et sur celui de la construction des savoirs.

Through the description of two (notably different) research programs dedicated to the inclusion of Oceanian languages in New Caledonia's diglossic context, the aim of this paper is to study the processes and implications of co-development when it comes to building knowledge. This comparison raises and enlightens many epistemological and ethical questions. We believe action research in education should be built as in cooperative approach gathering teachers in training, practicing teachers and researchers from various disciplinary fields. However, the relationship between research teams and teachers is still fragile (Nusbaum, 2008: 127). In addition, research implies an act of power as it constructs social realities and legitimizes them through scientific popularisation (Heller 2002) sometimes without everyone's consent. Action research that integrates interventions seems more efficient to us on both ethical values and the construction of knowledge. 


\section{INDEX}

Keywords : collaborative research, situated didactics, multilingualism, multiliteracy, coinvolvment

Mots-clés : recherche-collaborative, sociodidactique, plurilinguisme, pluri-littéracie, coimplication

\section{AUTEURS}

\section{CLAIRE COLOMBEL-TEUIRA}

Institut de Formation des maîtres de la Nouvelle-Calédonie, Eralo

Claire Colombel-Teuira est enseignante-chercheure et formatrice à l'institut de formation des maitres de la Nouvelle-Calédonie et membre associée à l'équipe Eralo de l'université de la Nouvelle-Calédonie. Ses travaux sociolinguistiques et sociodidactiques portent sur les thèmes des contacts de langues et du plurilinguisme, la contextualisation didactique, les langues objets et moyens d'enseignement et les interactions verbales.

colombel.claire[at]gmail.com

\section{VÉRONIQUE FILLOL}

Université de la Nouvelle-Calédonie, Eralo

Maître de conférences en sciences du langage (HDR) à l'Université de la Nouvelle-Calédonie, Véronique Fillol tente d'articuler l'analyse sociolinguistique à la didactique des langues-cultures. Ses travaux portent sur la description des plurilinguismes, la glottopolitique, les violences normatives, la didactique du français langue de scolarisation et la formation des enseignants en contexte plurilingue.

veronique.fillol[at]univ.nc 